\title{
BEHAVIOR OF STRESSES AND HYDRODYNAMICS FROM MULTICOMPONENT SEISMIC DATA
}

\author{
Boris P. Sibiryakov, Lourenildo W.B. Leite and Wildney W.S. Vieira
}

ABSTRACT. A method for fluid-stress modeling of 3D seismic, and drilling data, provided new information on the stress conditions, and on the hydrodynamics of the U11 layer of the Upper Jurassic sandstone reservoir in the Arigol field, Western Siberia, Russia. It is proposed to detect and outline oil fields, and divide them into isolated traps, marked by low sedimentary overburden pressure, and fluid-trapping properties based on correlation between the structural pattern and mapped stress. A different systematic approach was adopted, considering: the use of $3 \mathrm{D}$ component seismic data of $V_{\mathrm{p}}$ and $V_{\mathrm{s}}$ velocities; the density $\rho$, and the stress models of the reservoirs to detect the areas of low overburden pressure $P(x, y, z)$; and the vertical inclination of fractures (faults), $\varphi(x, y, z)$, caused by the non-hydrostatic behavior of stress.

Keywords: seismic structured media, porous media, fractured media.

RESUMO. Um novo método para a modelagem de tensão e fluidos em sísmica 3D, além de dados de perfuração, forneceram nova informação para as condições de tensão e para a hidrodinâmica da camada U11 do arenito reservatório do Jurássico Superior no Campo Arigol, Oeste da Sibéria, Rússia. São propostos métodos para a detecção e delimitação de campos de petróleo, e segmentá-los em trapas isoladas, marcadas por baixa pressão da sobrecarga sedimentar, e por propriedades de trapeamento de fluidos baseado na correlação entre os padrões estruturais e o mapeamento de tensões. Uma metodologia alternativa e sistemática foi adotada, considerando-se 0 uso de: dados sísmicos das componentes 3D para as velocidades $V_{\mathrm{p}}$ e $V_{\mathrm{s}}$; a densidade $\rho$, e a modelagem dos reservatórios para detectar as áreas de baixa pressão da sobrecarga $P(x, y, z)$; e a inclinação vertical de fraturas (falhas), $\varphi(x, y, z)$, causadas pelo comportamento não-hidrostático das tensões.

Palavras-chave: meios sísmicos estruturados, meios porosos, meios fraturados. 


\section{INTRODUCTION}

In the new fluid-stress model, contour lines of anomalous overburden pressure $(P)$ are used as hydrodynamic barriers for hydrocarbon migration, and the geometry of fluid flow lines (horizontal pressure gradients) corresponds to barriers between traps.

The areas of detected fluid-stress traps depend on the choice of the boundary values of constant overburden pressure and its horizontal gradients. It is proposed to map fluid-stress traps, and to predict their sizes in a way similar to that for structuraldepositional and structural-stratigraphic traps.

Deep drilling within the hydrodynamic screened traps should be undertaken, if they fall into the most contrasting and unexplored low pressure $(P)$ anomalies, aiming at to estimate the fluid-stress properties of the section.

Unlike the traditional methods of hydrodynamic modeling, the new method is based on 3D component seismic exploration data $\left(V_{\mathrm{p}}, V_{\mathrm{s}}, \rho\right)$. Stress, shear waves, porosity, specific surface, 3D hydrodynamic pressure modeling of buried oil and gas traps have received much attention recently.

This modeling is based primarily on downhole logging followed by the estimation of porosity and permeability with implications on the inner borehole space. In this approach, seismic data are used as supplementary information to specify the structural framework, and to find relationships between the seismic image and the hydrodynamic parameters of hydrocarbon reservoirs.

The present paper is part of a study about computing stresses and strains using $P$ and $S$ wave velocities in order to localize areas of small pressure in oil and gas producing layers as natural suction pumps, and it is devided in different relative independent parts.

The first part concerns conventional seismic investigations in order to obtain information about $\mathrm{P}$ and $\mathrm{S}$ wave velocities, and also the seismic boundary configurations. The second part concerns calculating stress and strain in the geological structures using the information obtained in the first part, and also the non-trivial behavior of pressure, since it can decrease with depth, and create natural pumps which accumulates fluids. And the third is concerned with calculating pressure discontinuities between solid and fluid, what depends on the structure of the pore space.

In order to start to predict stress and strain for real geological structures, we need to know $P$ and $S$ velocities, densities, and the seismic boundary configurations. This is a separate classical problem of seismic investigations. We restrict our present description to isotropic models, and for anisotropic cases the equations are more complicated.
It is mandatory that the acquired data be three component. From land observed data, we can use $S$ waves from horizontal vibroseis, together with VSP technology. From marine observed data, we can use AVO technology looking for converted P-S-P waves. In special cases, we can use petrophysical measurements of borehole data.

The first appearances on pore space and integral geometry were presented by Sibiryakov (2002) and Sibiryakov \& Prilous (2007). The theory of porous media is based on integral geometry because such mathematical discipline deals with collective geometrical properties of real reservoirs. It was shown by Santaló (1953) that such collective properties are namely for porosity, specific surface, average curvature and Gaussian curvature. For example, cracked media has, as a rule, small porosity, but very large specific surface area that creates anomalous high $\gamma=\frac{v_{S}}{v_{P}}$ ratio, what means a negative Poisson coefficient,

$$
\sigma=\frac{1-2 \gamma^{2}}{2-2 \gamma^{2}}
$$

The popular model of tubular pore space has a very high cross-sectional curvature, $K_{1}$, and a very small longitudinal curvature, $K_{1}$. This means that the Gaussian curvature $K_{\mathrm{G}}=$ $\sqrt{K_{1} K_{2}}$ is much less than average curvature $K_{\mathrm{A}}=\frac{K_{1}+K_{2}}{2}$. In granular media, $K_{\mathrm{G}}$ and $K_{\mathrm{A}}$ are close to each other (Smirnov, 1964).

\section{METHODOLOGY}

The general subject of the paper is a model for the calculation of fluid velocity prediction into borehole. This important study would be impossible without the prediction of stresses in the hydrocarbon (oil and gas) producing layer. After stress prediction, we must predict pressure discontinuities between the solid and liquid phases in order to calculate fluid pressure far from well, and then calculate the velocity of fluid into borehole.

The first research paper published on this subject was Sibiryakov et al. (2004), where percolating process was not considered. Besides, there was no words about optimal coordinates for well positioning. In the present work we continue with this study, and show all steps from common stress prediction, based on multicomponent seismic, to fluid velocity prediction using laboratory measurements of the pore space structure.

The state of elastic layered media is described in the general case by six (three normal and three tangential) stress tensor components. Complex stress behavior of buried traps is modeled on the basis of $\mathrm{P}$ and $\mathrm{S}$ layer velocities, and of the bulk density of reservoir and cap rocks. Overburden pressure and 
tangential stress intensity (the measure of non-hydrostatic behavior of stress) are other key parameters, the so-called scalar invariants of stress tensor. Overburden pressure is one third of the sum of vertical and horizontal stresses; if they are equal, the overburden pressure is simply the weight of the overlying (Sibiryakov, 2004).

Stress is non-hydrostatic even in horizontal layered media subject to only vertical gravity compactation without horizontal displacement. Vertical stress is defined as equal to the weight of the overburden in the form: $\sigma_{z z}=P_{z}=P_{0}$. The correspondent horizontal stress is sufficiently lower than $P_{z}$, and given by: $\sigma_{x x}=P_{x}=P_{0}\left(1-2 \gamma^{2}\right),\left(\sigma_{x x}=\sigma_{y y}\right.$ in this case, and applied in this paper example), where $\gamma=\frac{V_{S}}{V_{P}}$ (Sibiryakov et al., 2004).

Pressure is always a scalar quantity, and the simplest case for overburden pressure is

$$
P=\frac{1}{3}\left(\sigma_{x x}+\sigma_{y y}+\sigma_{z z}\right)=P_{0}\left(1-\frac{4}{3} \gamma^{2}\right),
$$

which is sufficiently less than the overlying weight $P_{z}$.

Structural effects complicate stress modeling, since in the general case the equilibrium equations should be integrated with the boundary conditions of loading continuity, displacement on layer boundaries, and zero stress on the free Earth's surface. However, overburden pressure depends on velocity ratio $(\gamma)$ and rock density.

Note that overburden pressure breaks at layer boundaries if the velocity ratio $\gamma$ has a jump. In the simplest case of layered media, the jump of overburden pressure at layer boundaries is $\Delta P=\frac{4}{3} P_{0}\left(\gamma_{1}^{2}-\gamma_{2}^{2}\right)$. Therefore, overburden pressure drops jumpwise with depth, if the underlying $\left(\gamma_{2}\right)$ velocity ratio is higher than the overlying $\left(\gamma_{1}\right)$ one. This idea would appear strange to geologists, but it is a fact related to the non-elementary behavior of stress in solids.

The behavior of continuous vertical stress at layer boundaries is rather simple, whereas horizontal stress behaves in a complex way and changes jumpwise, which causes the non-elementary behavior of overburden pressure as the sum of vertical and horizontal stresses. As a result, low pressure zones favorable for accumulation of fluids can arise even in the absence of anticlines. The presence of an anticline complicates the drop of overburden pressure, as rocks slide down the fold limbs produce additional horizontal extension, but this is not a necessary condition for the emergence of a favorable setting for fluid accumulation.

The intensity of tangential stress is a measure of mechanic instability, and it is the parameter responsible for the failure of the solid rock skeleton and fracturing. In simple layered media, tangential stress, scalar invariant, $P_{T}=J=\frac{1}{2}\left(\sigma_{z z}-\right.$ $\left.\sigma_{x x}\right)$, also depends on velocity ratio, and it is shown to be given by $J=\gamma^{2} P_{z}$. Obviously, tangential stress increases with depth and rock weight, which should cause fracturing and failure. Tangential stress breaks at layer boundaries if velocity ratio $\gamma$ differ in the different layers, and this discontinuity occurs also in the general case when tangential stress shows complex dependence on wave velocities and boundary geometry. The proportions between individual components of tangential stress and their intensities controls the inclination of fractures. Note that the above considerations concern primarily the present stress field. This is especially important in calculating fluid flow into boreholes, as the underground hydrodynamics is apparently controlled uniquely by the actual rock stress.

The key stress parameters of a section discontinuity (namely, overburden pressure, normal components of horizontal stress, intensity of tangential stress), for horizontal and non-horizontal boundaries, depend on the velocity ratio (Poisson's ratio) change at these boundaries. Therefore, multicomponent $P$ and $S$ wave seismic surveys are indispensable in studies of stress conditions of oil and gas traps. It does not mean that experiments should include both $\mathrm{P}$ and $\mathrm{S}$ surveys, but multicomponent VSP, multicomponent modifications of ultrasonic logging, or laboratory measurements of samples, can be used.

In addition, maps of stratigraphic surfaces, if they include the above stress parameters listed, should give two sets of data: one for the layer below, and the other for the layer above the mapped surface. Only three stress components are continuous in the general case, and the other three show jumpwise changes at layer boundaries.

Computing stress components implies integration of equations of equilibrium, that is a system that reflects the fact that the stress-induced 3D forces are equal to the gravity force (weight, overload, overload weight, $m g z$, mass $\times$ acceleration $\times$ thickness, stress dimension), whereas the horizontal forces cancel each other and their sum is zero.

The system of equations includes six unknown stresses and only three equations of equilibrium. Thus, it is not closed in the general case, but it can be made closed using an elastic model of stress-strain relationship. This is justified by relative simplicity of the model, and by the possibility to measure the elastic constants using P, S and PS waves. The closed system of partial derivative equations can be transformed into a system of integral singular equations. Methods for the transformation of these singular systems into regular ones were suggested by Kupradze (1963) and 
Parton \& Perlin (1985), and they imply in the use of conjugate operators of fundamental solutions. Sibiryakov \& Zaikin (1994) have applied this method for 2D applications to stress modeling in seismic sections in East and West Siberia.

However, direct extrapolation of the method for 3D problems complicates the computation. It can be simplified in view of the fact that many geological structures in West Siberia are quite shallow dipping; i.e., one tangential stress component is small relative to the two others. Sibiryakov \& Zaikin (1994) used a particular solution to inhomogeneous equilibrium equations as Poisson-type integrals where the integration is made over the structure volume. The effect of 3D structures on stress is more local than of 2D ones, as it decreases in inverse proportion to the square distance from the structure.

We propose here a new method to calculate Poisson-type curvature integrals that are reduced to a succession of $2 \mathrm{D}$ quadratures followed by common $1 \mathrm{D}$ integration. Stress is defined by velocity discontinuity and the effect of structure geometry.

As an example from the Arigol field, the local high (Vakh pattern holes) contribution due geometry is $20 \%$ of the total effects. Therefore, the overburden pressure in the producing bed, and in the layers below and above, is controlled mainly by the velocity ratio. The area has hundreds of wells, and the the overburden stress was estimated using density logs. The distribution of layer $P$ wave velocities was obtained by CMP stacks and VSP data from three boreholes. The greatest difficulty was in the estimation of velocity ratio, since there was no direct $\mathrm{P}$ or $\mathrm{S}$ velocity measurements made. Nevertheless, petrophysical data used ultrasonic measurements of the Jurassic sandstones, and allowed to find that the $\gamma$ ratio for the producing bed ranges between 0.577 and 0.550 . Velocity ratio for other layers were inferred from density-velocity relationships.

It is obviously expected that if the $\gamma$ ratio is higher in the producing bed, its overburden pressure is lower than in the overlying. Indeed, the pressure deficit in the producing bed is about 50 atmospheres in the average (Sibiryakov et al., 2004).

Figure 1 shows overburden pressure on the roof of a Jurassic reservoir. First, the pressures are about 30\% lower than in the overlying layer. Second, the pressure in the producing bed shows about to $5 \mathrm{MPa}$ drop at the transition to the overlying layer. The layer fluid pressure differs from the mapped overburden pressure.

The pressure discontinuity between the rock skeleton and the fluid is controlled by the structure of the pore space and by the skeleton-fluid compressibility ratio. However, the orthogonal trajectories, to the shown contour lines of overburden pressure, coincide with fluid flow lines, if the pore space structure in the producing bed remains invariable (Sibiryakov et al., 2004).

Examining the probable flow lines and calculated points of discharge in Figure 1, the local enclaves of these lines may account for depth variations of the oil-water contact (OWC). Therefore, orthogonal to pressure isolines on the surface of the producing bed are the probable fluid flow lines, and the sinks point to fluid and gas discharge. This fact should be taken into consideration as an additional criterion in the selection of new exploratory drilling sites.

Still referring to Figure 1, the overburden pressure and intensity of tangential stress estimated on the basis of velocity ratio $\gamma$ were assumed constant over the reservoir, and the flow lines are thus estimative. Almost all lines converge toward the top of the structure. The points of local discharge, to the right and left of the main borehole complex, are prominent even in this generalized model. More detailed information from S wave data may provide a basis for a higher-resolution map of the local discharge in the producing bed.

Figure 2 shows the map of tangential stress intensity for the same surface of Figure 1, where the region of lowest pressure almost coincides with faults within the region of minimum tangential stress, which indicates a certain stability of the structure as a whole. High tangential stresses cluster near a fault, which appears as a rather realistic result.

Maximum tangential stresses do not always coincide with faults detected by geological methods; possibly, because not all faults may be associated with the present-day stresses. This regularity can be used in age discrimination of faults.

Figure 3 shows calculated fracture inclinations, $\phi(x, y, z)$, around the structure. The fracture planes are inclined about 45 degrees with respect to the vertical, which may correspond to either randomly oriented fractures or to anisotropic medium with rhombic symmetry. However, fractures change their inclinations near faults to roughly vertical inclinations, thus producing another preferred inclination. This result can be checked by shear wave splitting techniques in multicomponent VSP.

The vertical inclination of fractures, called $\phi(x, y, z)$, are obtained from the rotation of the stress system to give the stress diagonal matrix, where the diagonal elements are the main stresses, and where $\sigma_{x x} \approx \sigma_{y y}$ (Novacky, 1970).

\section{Physical-mathematical theory}

We now follow with the physical-mathematical theory necessary to give a basement, and explain the results presented in Figures 1 , 2 and 3 , and we divided it into sections. 


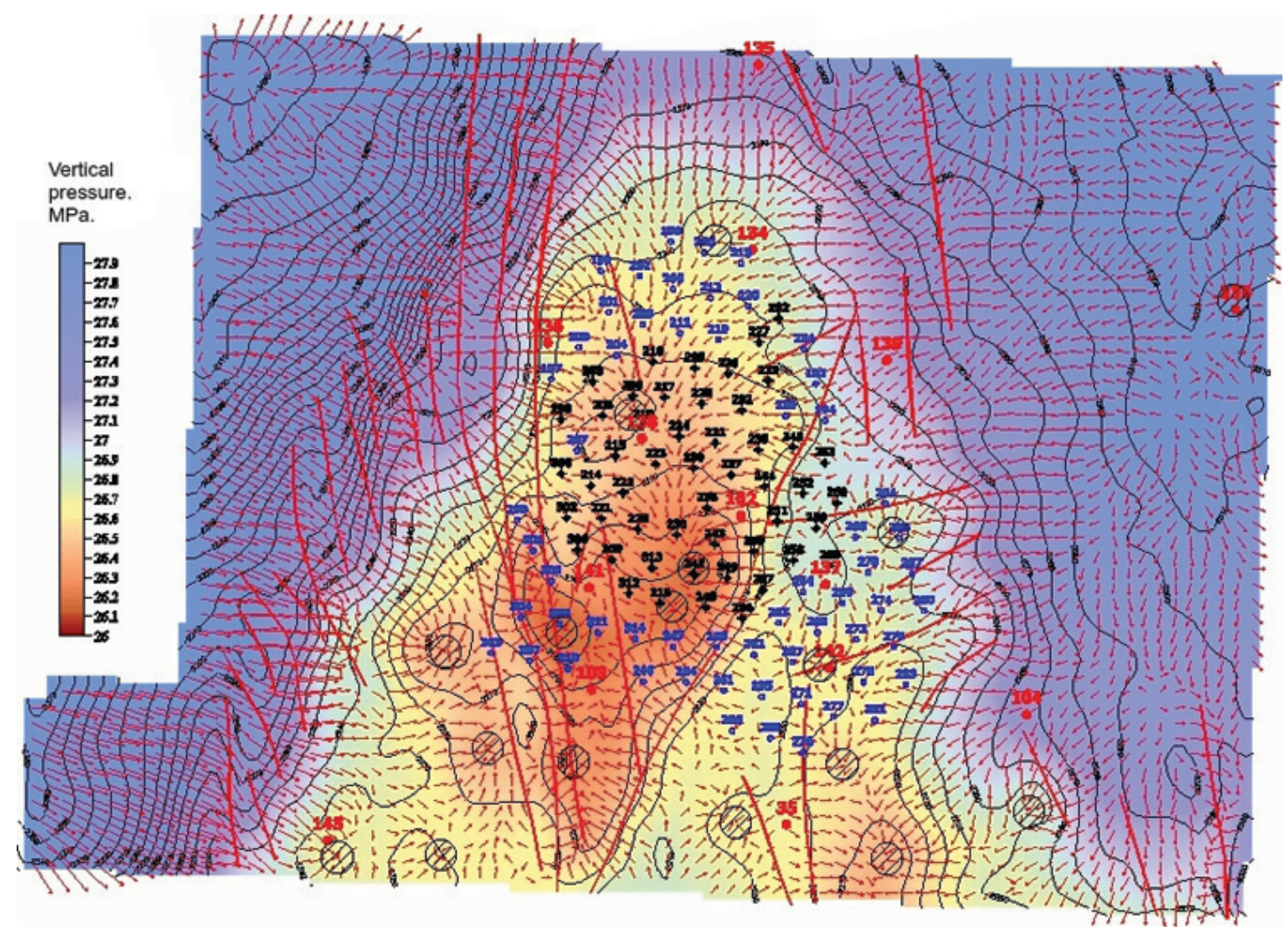

Figure 1 - Map of tectonic stress (represented by pressure $P$ ) of Jurassic reservoir, where the contour lines are overburden pressure $P=P_{0}\left(1-\frac{4}{3} \gamma^{2}\right)$ (dark blue lines) drawn in an arbitrary scale, and superposed to the color scale. Orthogonal trajectories are probable fluid flow lines (red arrows). The pressure scale is in color and placed on the left side, and shows variations between 26-28 Megapascal (MPa), $1 \mathrm{MPa}=10$ atmospheres. The center of the map is dominated by a low pressure zone. The dots with numbers are producing boreholes. Heavy red lines are geological faults. Isolated circular enclaves correspond to local hydrodynamic systems.

We start with the system of equations of equilibrium used to estimate stresses as given below, where two differential equations are homogeneous and one non-homogeneous (Sibiryakov \& Prilous, 2007):

$$
\begin{gathered}
\frac{\partial \sigma_{x x}}{\partial x}+\frac{\partial \sigma_{x y}}{\partial y}+\frac{\partial \sigma_{x z}}{\partial z}=0, \\
\frac{\partial \sigma_{y x}}{\partial x}+\frac{\partial \sigma_{y y}}{\partial y}+\frac{\partial \sigma_{y z}}{\partial z}=0, \\
\frac{\partial \sigma_{z x}}{\partial x}+\frac{\partial \sigma_{z y}}{\partial y}+\frac{\partial \sigma_{z z}}{\partial z}=\rho g .
\end{gathered}
$$

The first two Eqs. (1) and (2) correspond to full cancellation of horizontal 3D forces produced by inner stresses, and the last one means that vertical 3D forces are simply equal to the normalized gravity force.
The common solution of this system is to obtain the displacement components, and it is given by the sum of the fundamental solution, $u_{k}^{F}$, obtained for the complete homogeneous system, and of particular solution, $u_{k}^{P}$, given by the above nonhomogeneous system, in the form: $u_{k}=u_{k}^{F}+u_{k}^{P}$. To obtain this solution, it is used boundary integral methods. The result is a type of Poisson's integral, which illustrates the participation of additional displacement and stress, represented by volume $V$, due to more or less complex geometry of geological structures. The fundamental solution of the system of Eqs. (1), (2) and (3) is given by Novacky (1970) in the form:

$$
u_{k}(\mathbf{x})=\frac{g}{V_{S}^{2}} \int_{V} \Gamma_{k z}(\mathbf{x}, \mathbf{y}) d V_{y} .
$$

The additional displacement $u_{k}(\mathbf{x})$, due to structure deformation, is the integral of the fundamental solution, $\Gamma_{k z}(\mathbf{x}, \mathbf{y})$, over 


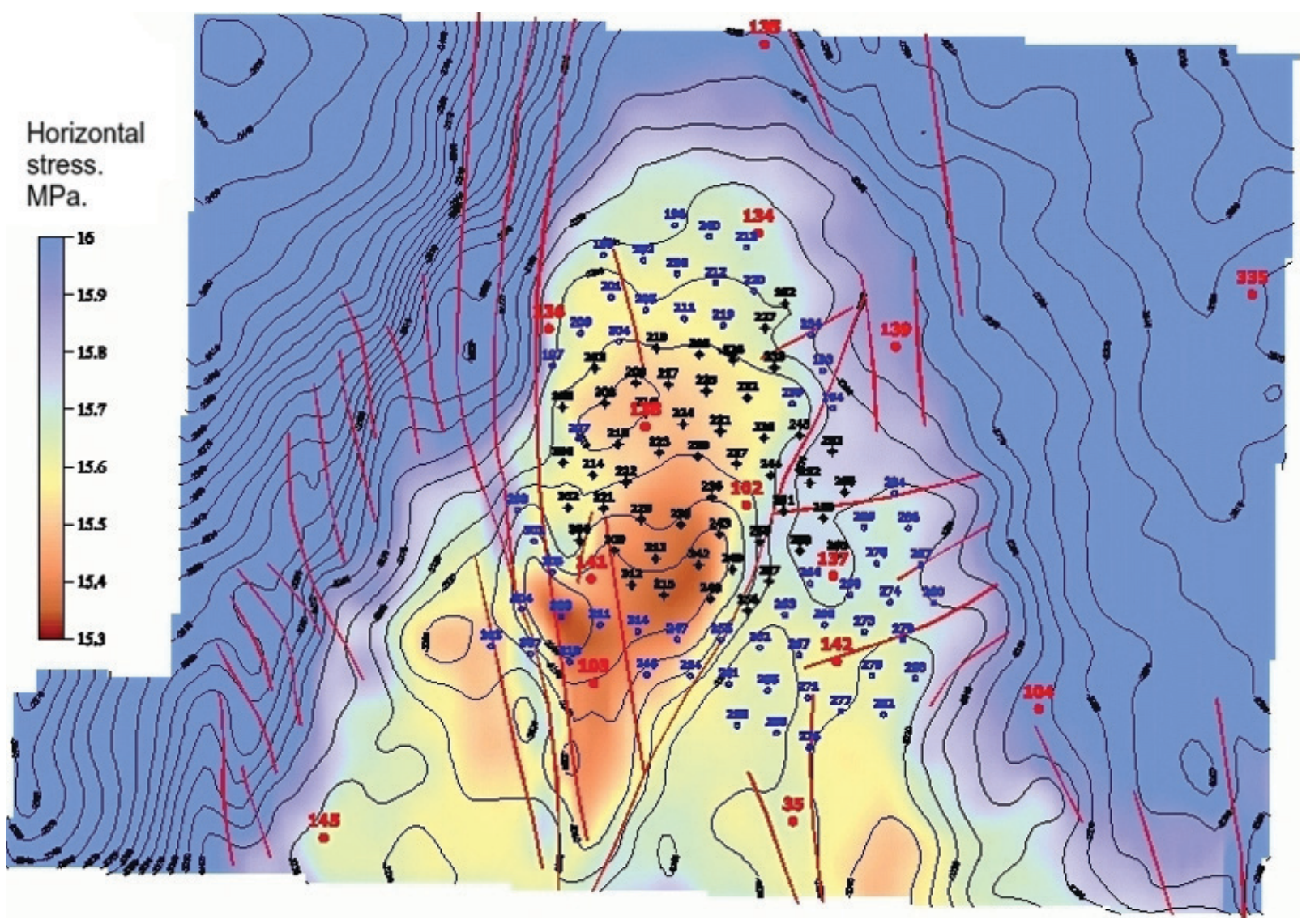

Figure 2 - Map of intensity of tangent stress, $J=\gamma^{2} P_{0}$, as a measure of non-hydrostatic pattern (dark blue lines), where the high values are located around small stress zones. The lines are drawn in an arbitrary scale, and superposed to the color scale. The color pressure scale is on the left side and shows values between 15.3-16.0 in MPa. The dots with numbers are producing wells. Heavy red lines are geological faults.

the structure volume $V$ of the equilibrium equation. It depends on the square $\mathrm{S}$ wave velocity, and $z$ in $\Gamma_{k z}$ relates to the vertical component, as in the gravity $g$. The integration covers all the volume represented by the $\mathbf{y}$ coordinate.

\section{Pressure discontinuity between rock skeleton and fluid}

Consider a fluid-filled reservoir, and let $u_{n}$ be the normal displacement of a grain skeleton. Applying the Gauss theorem, we obtain:

$$
\iint_{S} u_{n} d s=\iiint_{V} \operatorname{Div} \vec{u} d V=f V \frac{P_{0}}{\rho_{0} c^{2}}
$$

where $S$ is the grain surface, $V$ the total volume, $f V$ is the pore space volume, $P_{0}$ is the fluid pressure, and $\rho_{0} c^{2}$ is the fluid inverse compressibility (non-compressibility). Also,

$$
\iint_{S} u_{n} d s=\left\langle u_{n}\right\rangle S
$$

where $\left\langle u_{n}\right\rangle$ is the mean normal displacement, and by substituting this formula in Eq. (5) gives:

$$
\frac{P_{0}}{\rho_{0} c^{2}}=\frac{1}{f} \frac{\left\langle u_{n}\right\rangle}{r_{0}} \sigma_{0} r_{0},
$$

where $\sigma_{0}=\frac{S_{P}}{V_{T}}$ is the Specific Surface Area (SSP) $\left(S_{P}\right.$ is the total empty porous, and $V_{T}$ is the total volume of sample), and $r_{0}$ is the mean grain radius.

In a solid grain, the relation:

$$
\Delta\left(u_{i}+x_{i} e\right)=0
$$

is a rigorous relationship, where $\Delta$ is the Laplace operator, $u_{i}$ is the displacement in grain, and $e$ is the dilatation of the grain material. By definition, pressure is given by the dilation times the bulk module, as:

$$
P=e\left(\lambda+\frac{2}{3} \mu\right)
$$




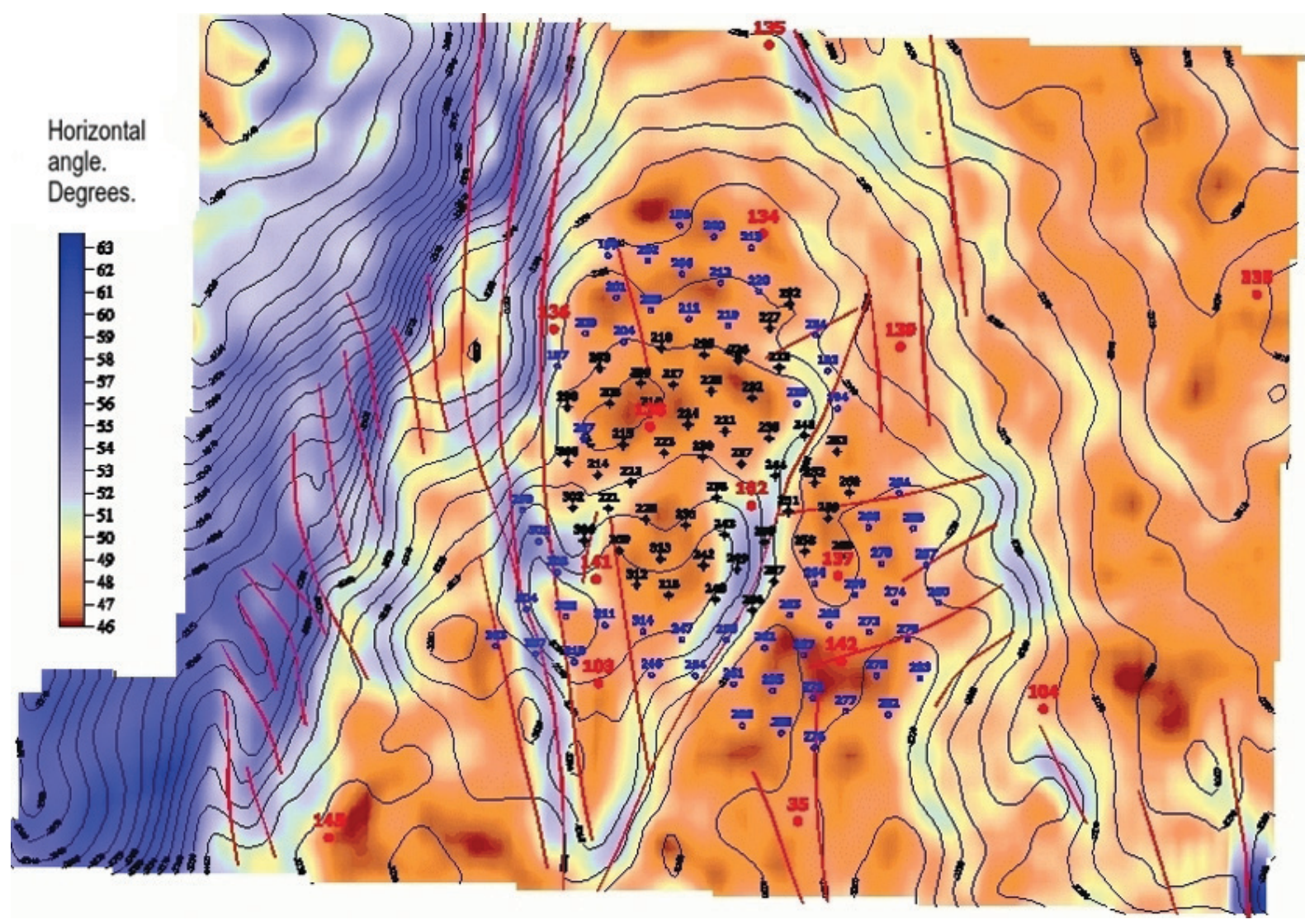

Figure 3 - Map of calculated fracture inclinations $\phi(x, y, z)$ in producing bed. The color scale of the left shows a variation between 46 and 64 degrees with respect to the vertical (to the left and/or to the right). The blue areas show roughly vertical fractures, and the brown areas show more normal fractures with a more chaotic distribution. In the center of the map, we can describe more randomly inclined fractures (darker areas). The dots with numbers are producing wells. Heavy red lines are geological faults.

Eq. (8) means, that the sum $u_{i}+x_{i} e$ is a harmonic vector. As its mean value is zero in the grain center, we can assume this harmonic function to be zero in the grain material, and make a relationship between displacement, $u_{i}$, and dilatation, $e$; in other words, $u_{i}=-x_{i} e$, and also for the average value in the form:

$$
\left\langle u_{n}\right\rangle=-\langle e\rangle r_{0} .
$$

Eq. (10) relates normal displacement in liquid and dilatation in solid.

Substituting the relations (10) and (9) into Eq. (7), we can write in explicit form the pressure discontinuity, $\frac{P_{0}}{P}$, between solid and liquid in the form:

$$
\frac{P_{0}}{P}=\frac{\sigma_{0} r_{0}}{f} \frac{\rho_{0} c^{2}}{\lambda+\frac{2}{3} \mu} .
$$

Eq. (11) shows that the pressure gradient in solid, $\nabla P$, perfectly coincides with the fluid flow lines if the pore structure, $\nabla P_{0}$, and it remains invariable in the producing layer, if the structure of the pore space (porosity and specific surface) are constant in this layer.

The pressure discontinuity depends on the ratio of two nondimensional parameters of the pore structure; to be specific, the product of the SSA $\left(\sigma_{0}\right)$ by the mean grain radius $\left(r_{0}\right)$ and inverse porosity $\left(f^{-1}\right)$. Yet, Eq. (11) contains small factors in the numerator and in the denominator which may lead to the instability of the estimation, especially in the case of a gas-saturated fluid.

Compressibility decreases abruptly at low gas contents, and viscosity varies slowly. Therefore, gas saturation of liquids is not necessarily favorable to oil production, but reduces the pressure gradient which is not always canceled by the respective fluid viscosity decrease.

According to Eq. (10), the problem of calculating fluid flow into a borehole is reduced to the integration of Poisson's equation:

$$
\Delta \dot{u}_{i}=\frac{1}{\eta} \frac{\sigma_{0} r_{0}}{f} \frac{\rho_{0} c^{2}}{\lambda+\frac{2}{3} \mu} \operatorname{Grad}_{i} P
$$


where $\operatorname{Grad}_{i}=\nabla_{i}=\frac{\partial}{\partial x_{i}},(i=x, y, z)$, and $\eta$ the fluid viscosity. Boundary conditions for this equation (conditions of viscous adhesion) require the velocities of particles be zero over the entire complex surface of pores. The problem of integration of Eq. (12) and flow rate estimation requires calculating of stress and, and also the knowledge of two parameters of the pore space and fluid compressibility.

The methods of integration for Eq. (12) are straightforward, even in the case of an arbitrarily complex pore structure; permeability is unnecessary as it appears in a complex way as a result of phase interaction and the pore structure. In a particular case of non-interacting fractures, modeled by rectilinear segments with constant crack opening, Eq. (12) can be easily integrated to yield the equation for flow rate in the form:

$$
\begin{aligned}
\dot{u}_{i} & =\frac{\delta^{2}}{12 \eta} \frac{\sigma_{0} r_{0}}{f} \frac{\rho_{0} c^{2}}{\lambda+\frac{2}{3} \mu} \operatorname{Grad}_{i} P \\
& =K \operatorname{Grad}_{i} P
\end{aligned}
$$

where $\delta$ is the mean crack opening, and $\delta^{2}$ represents permeability for this particular pore structure. Thus, the pressure difference between the skeleton and the fluid depends on the geometry of the pore space (mainly on its SSA), and on the hydrodynamic properties (compressibility and viscosity) of the fluid. The problem of the pore pressure estimation cannot be solved separately from the problem of general stress calculation. Eq. (13) plays the role of Darcy's law for the specific model now in focus.

\section{Percolation and permeability near borehole}

Sibiryakov (2002) described that the estimation of stresses and strains in oil-gas structure requires the integration of equations of equilibrium of the kind:

$$
\frac{\partial \sigma_{i k}}{\partial x_{k}}=\rho g, \quad(i, j, k=1,2,3 ; \text { or } x, y, z)
$$

for every layer.

For elastic rock deformation, the equation of equilibrium derived from Eqs. (1), (2), and (3) for the condition of Hooke's law, in closed form is given by:

$$
(\lambda+\mu) \operatorname{Grad}_{i} \operatorname{Div} \vec{u}+\mu \Delta u_{i}=\rho g_{i},
$$

where $g_{i},(i=x, y, z), g_{z}=g, g_{x}=g_{y}=0$ (Landau, 1988). The Lame parameters $\lambda$ and $\mu$ are determined from $P$ and $S$ wave velocities.

For boundary conditions, we can write the stress for the inside of any layer as an expression of the form:

$$
\begin{aligned}
& \sigma_{i k} n_{k}^{+}\left(S_{m}\right)=\sigma_{i k} n_{k}^{-}\left(S_{m}\right), \\
& u_{i}^{+}\left(S_{m}\right)=u_{i}^{-}\left(S_{m}\right),
\end{aligned}
$$

where $S_{m}$ stands for a surface numbered $m$, and $n^{+}$and $n^{-}$ points to outside or to the inside of the surface $S_{m}$.

On the free surface of Earth we have the evident stress boundary conditions in the form:

$$
\sigma_{i k} n_{k}^{+}\left(S_{0}\right)=0,
$$

where the summation over the repeated index is implicit.

Besides conditions in Eqs. (16) and (17), there are some conditions for the lateral surfaces that bounds the oil-gas structure. For the usual case, these conditions depend on the geological structures, but often these conditions are not sufficient, especially if the horizontal dimension of the structure is larger than the vertical dimension. Sometimes it is possible to use as boundary conditions the normal field of stresses without the effect of the structure.

The second part Eq. (16) becomes evident, because the fluid pressure equals the normal stress in solid matrix, and not the pressure in solid, because the lateral stresses are not equal to the normal stress.

To calculate the pressure discontinuities between liquid and solid, it is necessary to integrate the equation of equilibrium in a small volume of the producing layer with boundary conditions on the pore space like:

$$
\sigma_{i k} n_{k}^{+}(S)=P_{\infty}(S), \quad u_{i}^{+}(S)=u_{i}^{-}(S) .
$$

In the above equation, $P_{\infty}=$ constant is the pressure in the fluid. On the external boundaries of an elementary volume (edges of cube, for example) we require the same displacements like in the edges of elastic cube without the pore space. These conditions gives the constant value $P_{\infty}$, which is in equilibrium state with respect to the stresses in the solid. We have no doubt that this pressure depends on the structure of the pore space, but first of all on the SSA of the pore space.

The first step to attack the percolation and permeability is to calculate the stress for the entire area. The second part is to calculate the stress near the borehole. And the third step is to calculate the stress discontinuity between solid and liquid. As a result, we obtain the estimation of fluid flow as a function of the common stress-strain condition on the structure of the pore space, and on the fluid viscosity. 


\section{Volume dissipative forces by viscosity}

The tangential surface force, $F_{S \tau}$, due to viscosity is given by the expression $F_{S \tau}=\sigma_{0} \frac{\partial u_{\tau}}{\partial n}$, while the tangential volume force, $F_{V \tau}$, due to the same effect is given by:

$$
F_{V \tau}=\sigma_{0} \eta \frac{\partial u_{\tau}}{\partial n},
$$

where $\sigma_{0}$ is the SSA, $\eta$ is the viscosity, $\dot{u}$ is the particle velocity, and $n$ and $\tau$ are the normal and tangent directions of liquidgrain contact. For cracks sufficiently long compared to their opening, it would be convenient to use a simpler problem from the Navier-Stokes equation; namely, the solution of the problem about flowing between two infinite plates in the form (Landau, 1988):

$$
\frac{\partial p}{\partial x}=\eta \frac{\partial^{2} u_{\tau}}{\partial z^{2}} .
$$

In this equation $p$ is pressure in liquid, and $u_{\tau}=u_{x}=u_{x}(z)$ for the particular case of Figure 4, where $u_{y}=u_{z}=0$, and it represents a layout of the physical process of the flow, showing the sticking at the boundaries.

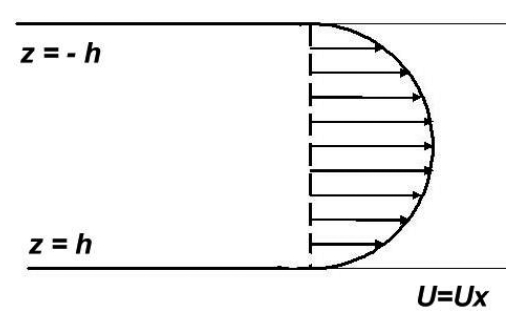

Figure 4 - Cross section of flow between infinite two plates. The length of dominant fracture is much larger than the crack opening.

The solution of Eq. (20) takes the form:

$$
\frac{\partial p}{\partial x}=P=\text { constant }, \quad \dot{u}_{x}=\frac{P}{2 \eta}\left(h^{2}-z^{2}\right) .
$$

In the above equations, $h$ is the crack opening. Hence, the surface force due to viscosity is given by the formula:

$$
\eta \frac{\partial p}{\partial x}=-h P \text {. }
$$

And the volume force acting in any point of the continuum is:

$$
\sigma_{0} \eta \frac{\partial p}{\partial x}=-\sigma_{0} h P=-\frac{2 \sigma_{0}}{h} \eta\left\langle u_{x}\right\rangle .
$$

In the above equation $\left\langle u_{x}\right\rangle$ is the average particle velocity between two planes. It means, that the Navier-Stokes equation contains an additional term that describes the volume dissipative force against the fluid velocity, namely:

$$
\left\langle F_{\tau}\right\rangle=-k^{2} \dot{u}_{r}, \quad\left(k^{2}=2 \frac{\sigma_{0}}{h}=\frac{\sigma_{0}^{2}}{f}\right),
$$

where $f$ is porosity.

\section{Stresses in solid near borehole}

For the solid near borehole the equations of equilibrium in cylindrical coordinates are:

$$
\begin{gathered}
\frac{\partial \sigma_{z z}}{\partial z}+\frac{\partial \tau_{r z}}{\partial z}+\frac{\tau_{r z}}{r}=\rho g \\
\frac{\partial \sigma_{r r}}{\partial r}+\frac{\sigma_{r r}-\sigma_{\varphi \varphi}}{r}+\frac{\partial \tau_{r z}}{\partial z}=0 .
\end{gathered}
$$

The correspondent pattern of stresses are shown in Figure 5.

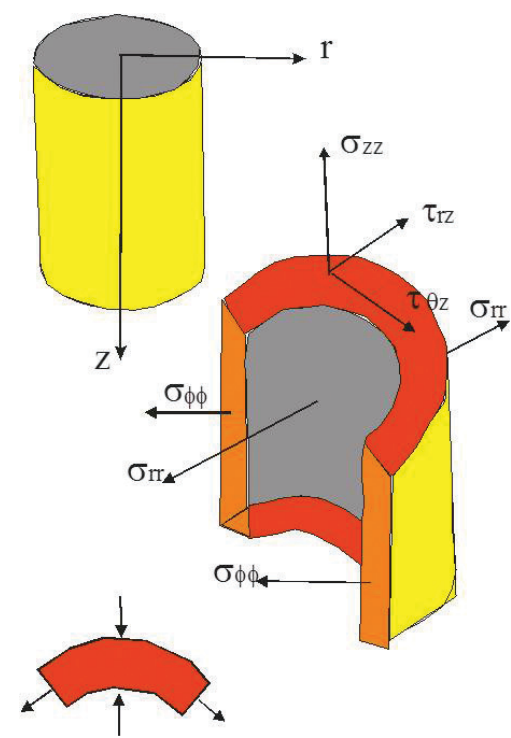

Figure $\mathbf{5}$ - Stress nomenclature in cylindrical coordinates, and the representation of the borehole with liquid and solid around it.

The above equations have very simple solutions, because for very large layer thickness in comparison to the radius of the borehole, the tangent stress $\tau_{r z}$ is equal to zero in all volume of rocks near the borehole.

There is an already known formula for the deformation $(e)$ of an elastic medium of density $\rho$, with a cylindrical cavity of radius $r_{0}$, and saturated by a liquid of density $\rho_{0}$, given by:

$$
e_{\varphi \varphi}\left(r_{0}\right)=\frac{u_{r}\left(r_{0}\right)}{r_{0}}=\rho g z\left(\frac{\sigma}{1-\sigma}-\frac{\rho_{0}}{\rho}\right) .
$$

In the above formula, $\sigma$ is the Poisson ratio, $u_{r}$ is the radial displacement, and $g$ the gravity. Besides that, considering that there is planar incompressibility (no change in density with respect to the $z$ coordinate, compression along $z$, dilation along $(x, y)$ axis, dilation along $z$ axis, compression along $(x, y)$ axis) in solid, we write for the deformation that:

$$
e_{r r}+e_{\varphi \varphi}=0
$$


The respective solutions of Eqs. (25) and (26) for the elastic stresses near borehole is given by the formulas (Sibiryakov, 1993):

$$
\begin{gathered}
\sigma_{r r}=\left(1-2 \gamma^{2}\right) P_{0}-\left(\frac{r_{0}}{r}\right)^{2} P_{0} \eta, \\
\sigma_{\varphi \varphi}=\left(1-2 \gamma^{2}\right) P_{0}+\left(\frac{r_{0}}{r}\right)^{2} P_{0} \eta .
\end{gathered}
$$

In Eqs. (29) and (30), $\gamma=\frac{V_{S}}{V_{P}}, P_{0}$ is the weight of overburden rocks, and $\eta=1-2 \gamma^{2}-\frac{\rho_{0}}{\rho}$.

\section{Equation of equilibrium and fluid velocity}

The equation of equilibrium with dissipative force around the borehole has the following form:

$$
\Delta \dot{u}_{r}-k^{2} \dot{u}_{r}-\frac{1}{\eta} \frac{\partial p}{\partial r}=0,
$$

where $\dot{u}_{r}$ is the particle fluid velocity in the borehole. The normalized components are: the first term $\left(\Delta \dot{u}_{r}\right)$ the volume force, the second $\left(-k^{2} \dot{u}_{r}\right)$ is the volume dissipative force, and the third $\left(-\frac{1}{\eta} \frac{\partial p}{\partial r}\right)$ the volume force due to pressure.

Let us try to find a solution for the above Eq. (31), considering that there is planar incompressibility for the liquid and for the solid, and that there is a linear relationship with respect to the vertical $z$ coordinate, like in Eq. (25). It means that there is a term $\frac{\partial^{2} \dot{u}}{\partial^{2} z^{2}}=0$ in the Laplace operator, such that this operator simplifies to the form:

$$
\Delta \dot{u}_{r}=\frac{\partial^{2} \dot{u}_{r}}{\partial r^{2}}+\frac{1}{r} \frac{\partial \dot{u}_{r}}{\partial r} .
$$

With the assumption of planar (not in volume) incompressibility, there is additional conditions for Eq. (32) in the forms:

$$
\frac{\partial \dot{u}_{r}}{\partial r}+\frac{\dot{u}_{r}}{r}=0, \quad \dot{u}_{r}=\frac{C(r)}{r^{2}},
$$

where $C(z)$ is an arbitrary function. Besides that, we can write Eq. (32) in the form:

$$
\begin{gathered}
\frac{\partial^{2} \dot{u}_{r}}{\partial r^{2}}+\frac{1}{r} \frac{\partial \dot{u}_{r}}{\partial r} \\
=\frac{\partial}{\partial r}\left(\frac{\partial \dot{u}_{r}}{\partial r}+\frac{\dot{u}_{r}}{r}\right)+\frac{\dot{u}_{r}}{r^{2}}=\frac{\dot{u}_{r}}{r^{2}} .
\end{gathered}
$$

Substituting Eq. (34) in Eq. (32) gives a simpler expression for Eq. (31) in the form:

$$
\frac{\partial p(r)}{\partial r}=\eta\left(\frac{C(z)}{r^{3}}-k^{2} \dot{u}_{r}\right) .
$$

Integrating the above equation with respect to $r$, with inferior limit $r$ and superior $R$, follows that:

$$
p(r)=P_{\infty}-\eta\left(\frac{C(z)}{2 r^{2}}-k^{2} C(z) \ln \frac{R}{r}\right) .
$$

To take into account, that on the borehole surface $r=r_{0}$, $p \rightarrow P_{0}, R>>r_{0}$, and $p(R)=P_{\infty}$ we get:

$$
\begin{gathered}
p\left(r_{0}\right)=P_{0} \\
=P_{\infty}-\eta\left(\frac{\dot{u}_{0}}{2 r_{0}}+k^{2} \dot{u}_{0} r_{0} \ln \frac{R}{r_{0}}\right) .
\end{gathered}
$$

In the above equation, $R$ is the horizontal range of producing layer, $P_{\infty}$ is the pressure in liquid outside the borehole (very far from it, and inside the reservatory), and $P_{0}$ is the pressure in borehole liquid.

From Eq. (37), we obtain the fluid velocity, $\dot{u}_{0}\left(r_{0}\right)$, into borehole in the form, and in its approximations:

$$
\begin{aligned}
\dot{u}_{0}\left(r_{0}\right) & =\frac{P_{\infty}-P_{0}}{\eta} \frac{2 r_{0}}{1+2 k^{2} r_{0}^{2} \ln \frac{R}{r_{0}}} \\
& \approx \frac{P_{\infty}-P_{0}}{\eta} \frac{1}{r_{0} \ln \frac{R}{r_{0}}} \frac{f}{\sigma_{0}^{2}} \\
& =K \frac{f}{\sigma_{0}^{2}},
\end{aligned}
$$

where

$$
K=\frac{P_{\infty}-P_{0}}{\eta} \frac{1}{r_{0} \ln \frac{R}{r_{0}}} .
$$

We simplified to the last form to emphasize the dependence of $\dot{u}_{0}\left(r_{0}\right)$ on $f, \sigma_{0}$, and its ratio $\frac{f}{\sigma_{0}^{2}}$, with $K$ representing a constant term. Figure 6 shows the plot of $\dot{u}_{0}\left(r_{0}\right)$ versus $\sigma_{0}$ and $f$, where the quantity $\frac{f}{\sigma_{0}^{2}}$ plays the role of permeability.

Also, for positive pressure contrast, $+\left|P_{\infty}-P_{0}\right|$, the flow is from layer reservatory to borehole, and when negative, $-\mid P_{\infty}-$ $P_{0} \mid$, from borehole to layer reservatory.

Due to large SSA parameter, the velocity is almost of zero flow in spite of sufficient porosity. It is interesting to mention that there is no necessity to use Darcy's law. Besides that, Eq. (38) does not contain the permeability coefficient, $k$. However, there is empiric relation between the SSA, $\sigma_{0}$, the porosity, $f$, and the permeability, $k$, namely (Sibiryakov \& Prilous, 2007):

$$
k=\frac{D_{p}^{2} f^{3}}{150(1-f)^{2}}, \quad D_{p}=\frac{6}{\sigma_{0}},
$$

where $S_{E}$ is the total area of the porous space, and $V_{T}$ is the total volume of the sample, and $\sigma_{0}=\frac{S_{E}}{V_{T}}$. The Eq. (39) can be rewritten as:

$$
\sigma_{0}=\frac{\sqrt{6}}{5} \frac{f^{\frac{3}{2}}}{(1-f)} \frac{1}{\sqrt{k}} .
$$




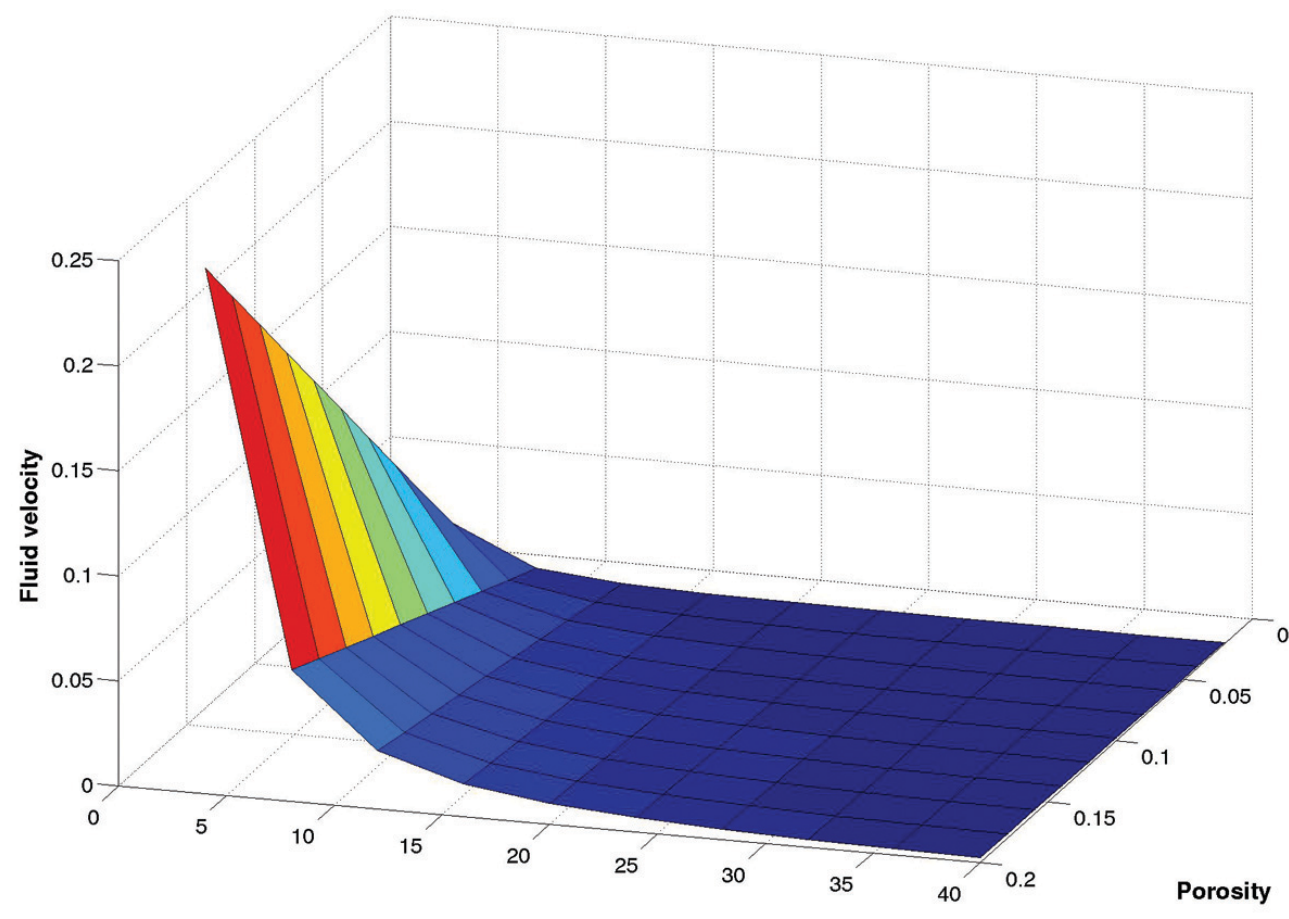

Specific surface area

Figure 6 - Plot of the Eq. (38) for the velocity flow as a function of porosity, $f$, and SSA, $\sigma_{0}$.

In spite of that, the percolation process depends on the porosity and on the SSA only. The hydrofracture effect is due to very small SSA for several large fractures compare to a lot of small fractures. Permeability plays the role of inverse square of the SSA.

\section{Estimation of liquid pressure far from borehole}

The value $P_{\infty}$ contained in Eq. (38) can be determined by solving the discontinuity problem existent between pressures of the solid and the liquid. The common stress-strain condition is given by integration of the equation of equilibrium Eq. (14), rewritten below:

$$
\frac{\partial \sigma_{i k}}{\partial x_{k}}=\rho g_{i}
$$

Let us take into account usual boundary conditions like continuity of force and displacement vectors. The configuration of boundaries and elastic properties are obtained by multiwave seismic.

The pressure is always a scalar. In liquid, the pressure, $P_{0}$, is defined as the normal stress in solid represented by $\sigma_{\mathrm{nn}}$. The pressure in solid, $P_{\mathrm{S}}$, is defined as the average of normal $\left(\sigma_{\mathrm{nn}}\right)$ and tangential stresses $\left(\sigma_{\tau_{i}}\right)$, in the form:

$$
P_{\mathrm{S}}=\frac{1}{3}\left(\sigma_{\mathrm{nn}}+\sigma_{\tau_{1}}+\sigma_{\tau_{2}}\right) \text {. }
$$

For simply common stress conditions with the vertical component equal to the weight of rocks, and the horizontal components containing a factor of the type $\frac{\sigma}{1-\sigma},(\sigma$ is the Poisson coefficient), it is possible to formulate the following problem related to Figure 7.

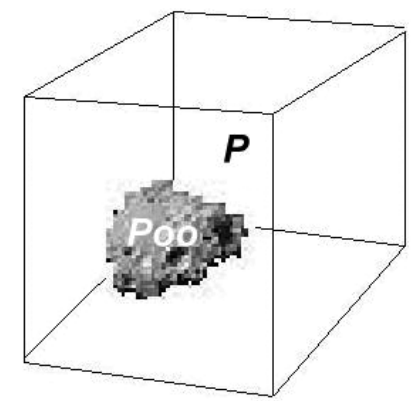

Figure 7 - A cube volume where the liquid pressure is $P_{\infty}$ and in the solid is $P$.

The elementary cube is submitted to vertical displacement $u_{0}$, while horizontal displacements are equal to zero. Inside the cube there is a liquid with constant pressure $P_{\infty}$. On the boundary solid-liquid the average normal displacement is given by the formula:

$$
\left\langle u_{n}\right\rangle=\frac{P_{\infty}}{\rho_{0} c_{0}^{2}} \frac{f}{\sigma_{0}} .
$$


Under the condition of fluid incompressibility, then for the average tangent $\left(u_{S}\right)$ displacement on the boundary solid-liquid it can be shown that:

$$
\left\langle\frac{\partial u_{S}}{\partial n}\right\rangle=-\frac{\left\langle u_{n}\right\rangle}{\pi l_{0}}
$$

with $l_{0}$ as a dominant distance between cracks. This result comes from differential geometry, and the quantities $\left\langle\frac{\partial u_{S}}{\partial n}\right\rangle$ and $\frac{\left\langle u_{n}\right\rangle}{\pi l_{0}}$ have opposite sign due to physical counter action at the boundary solid-liquid.

The relation between $\sigma_{0}, l_{0}$ and $f$ is described by Sibiryakov \& Prilous (2007), and it has a simply form given by:

$$
\sigma_{0} l_{0}=4(1-f)
$$

The simplification of boundary conditions as in Eqs. (42) and (43) gives the possibility to determine all stresses and displacements as a function of the unknown constant $P_{\infty}$. On the vertical sides of the cube there are displacements equal to constant value, but on the other side it is equal to zero. The usual boundary condition; to be specific, equality between normal force in solid and unknown pressure $P_{\infty}$ in liquid, gives a functional equation for determining the value $P_{\infty}$ as an inverse problem, namely:

$$
\left\langle\lambda \theta\left(P_{\infty}\right)+2 \mu e_{n n}\left(P_{\infty}\right)\right\rangle=P_{\infty},
$$

for given $\theta\left(P_{\infty}\right)$ and $e_{n n}\left(P_{\infty}\right)$ functionals, with $\lambda$ and $\mu$ as Lame parameters of the solid material.

\section{CONCLUSIONS}

We described the main steps for the estimation of complex stress behavior in a given structural-velocity model of a medium, and calculation of the storage capacity of buried traps. The first results of mathematical modeling and geological interpretation of the obtained fluid-stress maps are associated with the formation of a new step in applied geodynamics, namely fluid-stress modeling of oil and gas traps.

The basic principles of this modeling are as follows. The relationship between overburden pressure, which is a scalar function $P(x, y, z)$, and rock stress (six components in the 3D model: $\left.\sigma_{x x}, \sigma_{y y}, \sigma_{z z}, \sigma_{x y}, \sigma_{x z}, \sigma_{y z}\right)$ is a fairly complex function in a general case. However, it is obvious that fluids accumulate in closed sites of porous reservoirs where the overburden pressure is minimum.

Under these assumptions, detected regions are a sort of fluid-stress traps in which hydrocarbons can accumulate rather by influence of stress ( $\sigma=$ constant) than by structural $(z=$ constant) factors.
Maps of horizontal gradients of overburden pressure are especially informative for the detection and outlying these traps, and they reveal: convergent (inflow) and divergent (outflow) pressure gradients; isolated inflow regions in the detected trap separated by the geometry of outflow lines (fluid-stress barriers); and possible fluid-stress traps in structural and non-structural conditions.

There is no possibility to use the Biot parameters, and the reason is that we have seismic waves velocities and densities of rocks, what gives a possibility to calculate Lame's constants for the integration of equilibrium equations.

The construction of the maps of Figures 1 and 3 used log data, core samples and 3D seismic data image of the Upper Jurassic sandstone reservoir of the Arigol field, that contains numerous sites of lithological replacement and faulting. Geological prospecting surveys show different depths of oil-water contact (OWC), what contradict the accepted arch-layer model for the reservoir.

The paradoxical behavior of the OWC depths finds a sound explanation within the limits of a fluid-stress model, since the single Upper Jurassic reservoir was subdivided by us into nine fluidstress traps on the basis of correlation of the overburden pressure and its horizontal gradients (see Fig. 2).

Comparison of the maps of intensity of tangential stresses $J(x, y, z)=\frac{1}{2}\left(\sigma_{z z}-\sigma_{x x}\right)=\gamma^{2} \sigma_{z z}$ and of inclinations $\phi(x, y, z)$ of stress-produced fractures (see Figs. 2 and 3 ) leads to the following conclusions:

- faults detected from 3D seismic data correspond to local anomalies of tangential stresses;

- the observed mismatch can be related to the difference between the present-day stress and the past stress along the faults;

- different OWC depths in boreholes with numbers 124, 136, 139 can be caused by their position in different fluid-stress zones.

At this point, an interesting question arises of how to separate tangential stress (all stress out the orthogonal plane) in rock matrix from their natural fractures, since seismic data do not allow seeing in this centimeters scale (sub-seismic data). The answer is related to the scalar $J(x, y, z)$ that is responsible to produce cracks, if it is sufficiently large, and we can only calculate the probabilistic orientation of cracks.

Note that the method of fluid-stress modeling has some limitation as quantitative estimates of stresses are, both in oilsaturated and water-saturated, as well as dry rocks. Therefore, 
it must always be integrated with other methods of seismicgeological modeling. At present, the fluid percolation theory based on Darcy's law says that we can ignore stress-strain state in solids; besides, percolation theory contains porosity, and does not contain SSA that creates forces to stop percolation.

We need predict stress-strain in solid, and pressure discontinuity between phases, what depends on the structure of the pore space, and not only on porosity. Therefore, it is not necessary to use Darcy's law for determining the permeability, since it is only a geometric property of porous medium. Permeability value is directly proportional to porosity, and inversely proportional to the square of the specific surface for a specimen.

Porosity and SSA give a possibility to use alternative methods for measuring permeability.

The velocity of fluid into a borehole depends on common stress-strain conditions of the producing layer, on the structure of the pore space (SSA and porosity) and on the viscosity of the fluid.

The solution shows that the main factor of oil production is the specific surface area of porous and cracks. The solution does not contain the permeability; instead, there is the inverse square of the SSA that plays the role of permeability.

The hydrofracture effect is due to a very small SSA for several large fractures, in comparison to a lot of small fractures.

\section{ACKNOWLEDGEMENTS}

The authors would like first to thank the paper reviewers for their patience and positive contribution. We would like to thank the Brazilian institutions UFPA (Universidade Federal do Pará), FINEP (Financiadora de Estudos e Projetos) and PETROBRAS (Petróleo Brasileiro S/A) for the research support. Extended thanks are to the Project National Institute of Science and Technology (Instituto Nacional de Ciência e Tecnologia), INCT-GP, MCT/CNPq/FINEP. The authors thank specially to the Project Science Without Borders of CNPq/CAPES of the Brazilian Federal
Government that sponsors this research aiming at oil exploration.

\section{REFERENCES}

KUPRADZE WD. 1963. Potential methods in elastic theory (in Russian). [S.I.]: Issues of Physics and Mathematics. Moscow, Russia. 472 pp.

LANDAU LD. 1988. Hydrodynamics (in Russian). [S.I.]: Nauka. Moscow, Russia. 165 pp.

NOVACKY W. 1970. Elasticity Theory. [S.I.]: Warsaw. 872 pp.

PARTON VZ \& PERLIN PI. 1985. Mathematical Methods of the Theory of Elasticity. [S.I.]: Imported Pubn. 673 pp.

SANTALÓ L. 1953. Introduction to integral geometry. Hermann et Cie, Editors. Paris, France. 123 pp.

SIBIRYAKOV BP. 1993. The stress-strain situation of rocks in vicinity of borehole. Russian Geology and Geophysics, 34(6): 73-80.

SIBIRYAKOV BP. 2002. Supersonic and intersonic cracking in rock-like material under remote stresses. Theoretical and Applied Fracture Mechanics, 38(3): 255-265.

SIBIRYAKOV BP. 2004. Dynamics of microinhomogeneous geological media. [S.I.]: Novosibirsk State University, Russia. 234 pp.

SIBIRYAKOV BP \& PRILOUS BI. 2007. The unusual small wave velocities in structural bodies and instability of pore or cracked media by small vibration. WSEAS Transactions on Applied and Theoretical Mechanics, 7(2): 139-144, July.

SIBIRYAKOV BP \& ZAIKIN AD. 1994. Multiple seismics and applied geodynamics in petroleum-bearing regions. Russian Geology and Geophysics, 35(5): 49-55.

SIBIRYAKOV BP, SIBIRYAKOV EB, GLEBOV AF, NESTEROV VN \& SOKOLOV EP. 2004. Prediction of stress and hydrodynamic behavior of fluids from multicomponent seismic data. Russian Geology and Geophysics, 45(6): 752-759.

SMIRNOV VI. 1964. Course of Higher Mathematics. London: Pergamon Press. $630 \mathrm{pp}$. 


\section{NOTES ABOUT THE AUTHORS}

Boris Petrovich Sibiryakov is a Special Professor of Geophysics in the Russian Academy of Sciences (RAS), Novosibirsk Branch, and in the University of Novosibirsk. At present is a Special Visiting Professor at the Geophysics Graduate Course of the Universidade Federal do Pará (UFPA), Brazil, participating in the Project Science Without Borders of CAPES/CNPq. Obtained the Masters and the Doctorate degrees in Geophysics from the University of Saint Petersburg, and the Doctorate from the Academy of Sciences, Novosibirsk. Develops research in applied seismic of fractured media for oil and gas exploration.

Lourenildo Williame Barbosa Leite is a Professor of Geophysics in the Undergraduate and Graduate Courses of the Universidade Federal do Pará (UFPA), Brazil. Obtained the Masters and the Doctorate degrees in Geophysics from Saint Louis University, Missouri, USA. Develops research work in applied seismic for oil and gas exploration aiming at velocity analysis, stack, inversion and migration. Is a member of SBGf, SEG, EAGE and SSA scientific societies.

Wildney Wallacy da Silva Vieira is a Doctorate student at the Graduate Course in Geophysics of the Universidade Federal do Pará (UFPA), Brazil. Obtained the Bachelors degree in Mathematics (in 2007), and the Masters of Geophysics (in 2011), both degrees at UFPA. Develops research work in seismic methods for oil and gas exploration. Area of interest has been ultimately focused at imaging by a complete processing workflow passing by data quality control, velocity analysis, inversion and migration. 